\title{
Infectious endocarditis: unusual cause of lower back pain
}

\begin{abstract}
Endocarditis has been shown to be a common cause of vertebral osteomyelitis. This combination is potentially fatal and needs to be diagnosed early with an echocardiogram. Suspicion is the key, with a complete history taking and physical examination. In this case, our objective was to report the late diagnosis of endocarditis in one patient with spondylodiscitis caused by Staphylococcus aureus, community-acquired. Imaging tests showed an osteolytic lesion of the lumbar vertebrae that after the investigation was defined to be due to complications of bacterial endocarditis. Concerning the duration of treatment and hospitalization, there is no significant difference between IE alone and IE with spondylodiscitis, especially with regard to patient age and gender, clinical and laboratory features, and evolution of the disease.
\end{abstract}

Volume IO Issue I - 2020

\author{
Ivan Cadena Vélez, Manuela Grego, Luis Siopa \\ Internal Medicine Department, Hospital Distrital de Santarém, \\ Portugal
}

\begin{abstract}
Correspondence: Ivan Cadena Vélez, Internal Medicine Department, Hospital Distrital de Santarém, Portugal, Tel +579|4040788, Email icadenavelez@gmail.com
\end{abstract}

Received: January 14,2020 | Published: January 22, 2020

Keywords: endocarditis, infectious, staphylococcus, spondylodiscitis

\section{Introduction}

Infective endocarditis is a relatively uncommon but potentially lethal disease. Although medical and surgical therapies have contributed to ameliorating prognosis, the mortality rate remains high. Presenting symptoms are variable and may sometimes delay the diagnosis. Musculoskeletal manifestations are reported frequently, and they can represent the major sign of the disease, leading to diagnostic difficulties. Septic vertebral locations during infective endocarditis are unusual; this association accounts for only a small percentage of the rheumatologic forms of infective endocarditis (IE), early diagnosis, and appropriate treatment to reduce complications. Thus, in patients with signs and symptoms of discitis, the investigation of cardiac valve involvement is relevant.

\section{Case report}

A 78-year-old woman with a history of a biological aortic prosthesis, implanted in 2016, and atrial fibrillation was admitted to the emergency department due to progressive worsening of low back pain for 3 weeks with irradiation to the lower limbs and severe gait limitation. The pain was poorly responsive to analgesics. She had no history of trauma. At the physical examination, she presented apyretic, tachycardic, sleepy, with degree III/VI systolic murmur without irradiation and palpation pain in thorny apophyses in D12 to L4. No sensory or motor deficit. Blood analyses revealed $\mathrm{Hb}: 9.9 \mathrm{mg} / \mathrm{dL}$, leukocytosis 10,800 with neutrophilia $83 \%$, Protein-C-reactive $11 \mathrm{mg} / \mathrm{dL}$, VSG: $131 \mathrm{~mm} / \mathrm{h}, \mathrm{Cr}$ : $2.4 \mathrm{mg} / \mathrm{dL}$, Urea $99.7 \mathrm{mg} / \mathrm{dL}$; LDH 313, CK: 506. Lumbar CT scan revealed "multiple and small millimetric osteolytic lesions in almost all of the lumbar somatic bodies and the left lateral slope of the upper S1 platform, some with rupture of the cortical bone." After hospital admission, there was a worsening of the general state of the patient with sudden onset of fever, tachycardia, and exacerbation of severe intensity low back pain associated with paraparesis and Janeway cutaneous lesions in the plantar region of the foot (Figure 1). There were no signs of acute heart failure. She performed an ECG with atrial fibrillation and a CT-EC without acute ischemic changes. Due to the high suspicion of endocarditis, blood cultures were collected, and a transesophageal echocardiogram was performed, revealing vegetation on the ventricular side of the biological prosthesis in aortic position without obstruction and without associated regurgitation or local complications (Figure 2). Given the new findings, it empirical antibiotic therapy with vancomycin and gentamicin was initiated and a lumbar spine magnetic resonance imaging was performed that showed "D12-L1 reduction in inter somatic height, vertebral platform irregularities, disc edema and diffuse alteration of the bone matrix signal of the D12 and L1 vertebral bodies (T2/STIR and hypoT1) with marked enhancement, translating inflammatory/infectious changes in the context of spondylodiscitis" (Figure 3) Hemocultures were positive for $\mathrm{S}$. epidermidis methicillin-resistant.

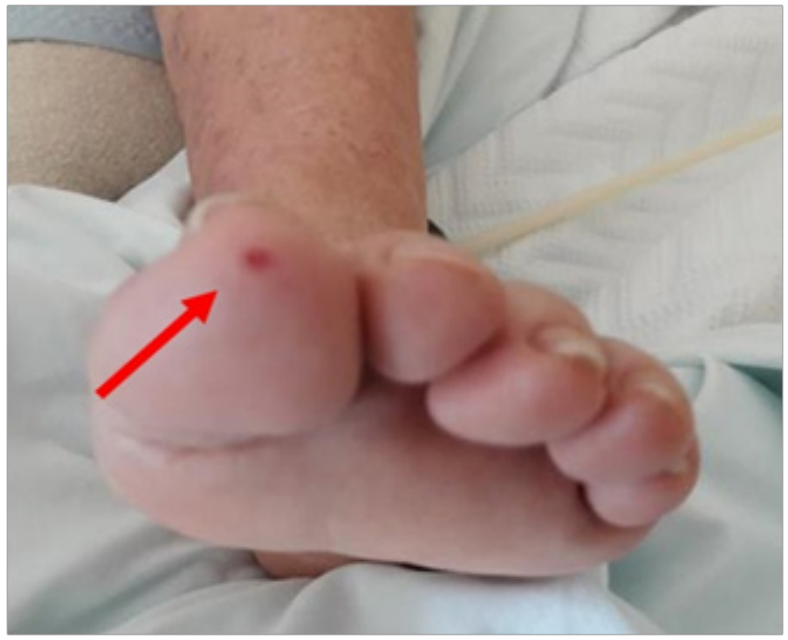

Figure I Janeway cutaneous lesion.

The patient was then admitted to the cardiology department, where she completed a total of 6 weeks of targeted antibiotic therapy with vancomycin and rifampicin after the acquisition of negative blood cultures with good clinical and analytical improvement. At that time, she was discharged and completed the 12-week antibiotic period stipulated for spondylodiscitis with linezolid in an outpatient setting. ${ }^{1}$ 


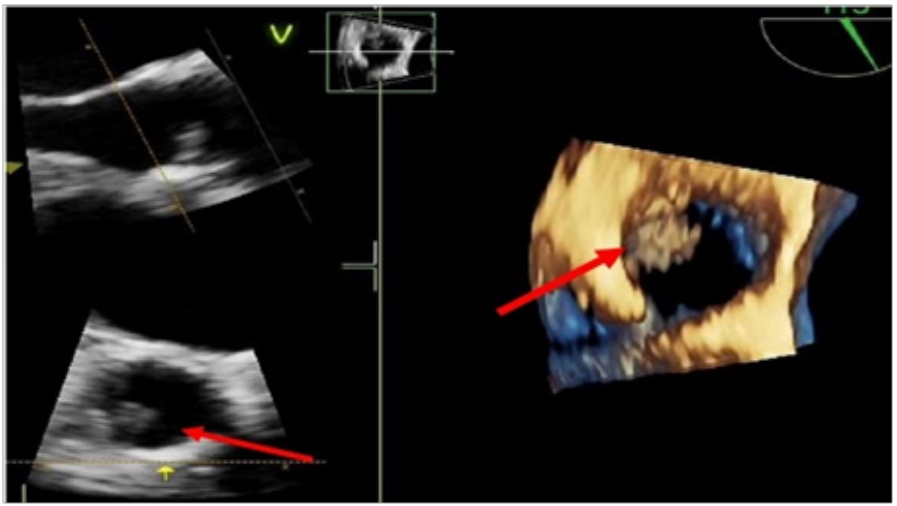

Figure 2 ETE:Vegetation on the ventricular side of the biological prosthesisin aortic posicion.

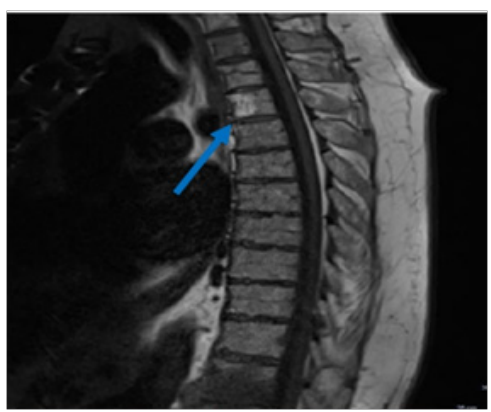

Figure 3 Inflamatory changes in the contex of spondylodiscitis.

\section{Discussion}

Previously reported percentages of the association vary from 5 to $13 \%$, but the rarity of this association contrasts with the reported high frequency of back pain in patients with IE. ${ }^{2.3}$ The apparent rarity of spondylodiscitis with IE contrasts with the higher frequency of back pain in patients with IE. In most studies of IE, back pain was present in up to $43 \%$ of patients. Localized pain was always present and antedated the diagnosis of IE by many days (an average of 22 days). The classical presentation of patients is a history of back pain associated with asthenia and pyrexia, although none of these symptoms are specific. The management of any patient with a history of uncontrollable back pain should include taking a detailed history and inquiring about the nature of the pain, as well as its exact location, presence of radiation from the painful site, and exacerbating and relieving factors. It is crucial to enquire about the past medical history of patients, noting that comorbidities such as cancer, diabetes, steroid medications, and chronic renal or hepatic diseases can predispose one to spondylodiscitis. ${ }^{4}$ Other factors such as smoking, alcohol, asthma, and poor dental hygiene have all been shown to be predisposing factors for infection. ${ }^{5}$

As regards the pathogenesis, some author has suggested that back pain is due to microemboli of immune complexes with or without bacteria. ${ }^{3.6}$ From a microbiologic point of view, a distinctive sign between IE 1 associated to spondylodiscitis and spondylodiscitis alone maybe the specific infective agent: a large predominance of S. viridans and other kinds of streptococci are found in patients with IE alone or with IE 1 spondylodiscitis, whereas a staphylococcus species usually is identified in most patients with spondylodiscitis is alone when a bacterial growth is obtained. ${ }^{6}$
It is important to note that among patients with a diagnosis of discitis, those with neurological manifestations such as paraparesis, paraplegia, and meningitis are more likely to have tuberculosis or staphylococcus infections. The examination should include a full neurological examination, a head-to-toe inspection looking for a possible site of entry (ulcers, pressure sores, furuncles, abscesses, recent wounds), and a chest examination to exclude chest infections or a heart murmur.

In terms of the duration of treatment and hospitalization, there is no significant difference between IE alone and IE with spondylodiscitis, especially with regard to patient age and gender, clinical and laboratory features, and evolution of the disease. That suggests that the occurrence of spondylodiscitis in a patient with IE does not worsen the patient's prognosis. Rather, the outcome depends mainly on the severity of IE..$^{7-8}$

Data from the literature and from the present study underline the importance of a preexisting heart valve disease as an important indication to search for IE as the source of the spondylodiscitis. ${ }^{9-10}$ In spondylodiscitis associated with IE, the mortality rate is still relatively high, whereas, in spondylodiscitis alone, it is almost zero. ${ }^{10}$ Therefore, it seems very important to exclude the diagnosis of IE in all patients with spondylodiscitis, considering that clinical and radiologic patterns are similar. Echocardiograms and blood cultures should be routinely performed in these patients.

\section{Conclusion}

Our case and the literature highlight the fact that endocarditis is a frequent cause of spondylodiscitis (up to $30 \%$ in certain series). Complications of this condition, if not diagnosed, can be fatal, and it is crucial to keep in mind that a complete medical history and chest examination and infrequent signs are a crucial part of the working diagnosis in these patients. If in doubt, cheap, easy, non-invasive echocardiography will reassure the clinician and rule out endocarditis as a cause of infection.

\section{Conflicts of interest}

The authors declare that they have no conflict of interest regarding this article.

\section{Sources of funding}

The authors declare that they have not received subsidies or scholarships for the elaboration of the article.

\section{References}

1. Churchill MA, Geradi JE, Hunder GG. Musculoskeletal manifestations of bacterial endocarditis. Ann Intern Med. 1977;87:754-759.

2. Hacene A, Flipo RM, Deprez X, et al. Endocardites et spondylodiscitis infetieuses. Rev Rhum Engl Ed. 1996;63:632.

3. Härkönen M, Olin PE, Wennström J. Severe backache as a presenting sign of bacterial endocarditis. Acta Med Scand.1981;210:329-331.

4. Carragee E. Pyogenic vertebral osteomyelitis. J Bone Joint Surg Am. 1997;79(6):874-879.

5. Mulleman D, Philippe P, Senneville E, et al. Streptococcal and enterococcal spondylodiscitis. High incidence of infective endocarditis in 50 cases. $J$ Rheumatol. 2006;33(1):91-97. 
6. Ninet J, Gayet HL, Etienne J, et al. Bacterial endocarditis presenting as acute vertebral osteomyelitis: 14 cases. Eur Heart J. 1984;5(Suppl C9):101-105.

7. Le Moal G, Roblot F, Paccalin M, et al. Clinical and laboratory characteristics of infective endocarditis when associated with spondylodiscitis. Eur J Clin Microbiol Infect Dis. 2002;21:671-675.

8. McHenry MC, Easley KA, Locker GA. Vertebral osteomyelitis: long term outcome for 253 patients from 7 Cleveland-garea hospitals. Clin Infect Dis. 2002;34(10):1342-g1350.
9. Jacquemart $\mathrm{T}$, Houvenagel $\mathrm{E}$, Buisset $\mathrm{H}$, et al. Spondylodisciterévélatriced'uneendocarditebactérienne. A proposd'une observation. Revue de la littérature. Ann Cardiol Angeiol. 1989;38:209213.

10. Pascaretti C, Legrand E, Laporte $J$, et al. Les endocarditis bactériennesrévélées par unespondylodiscite infectieuse. Rev Rhum. 1996;63:129-133. 